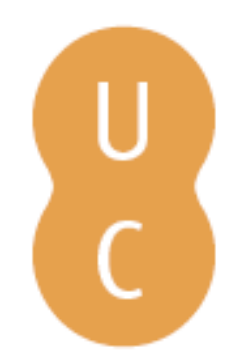

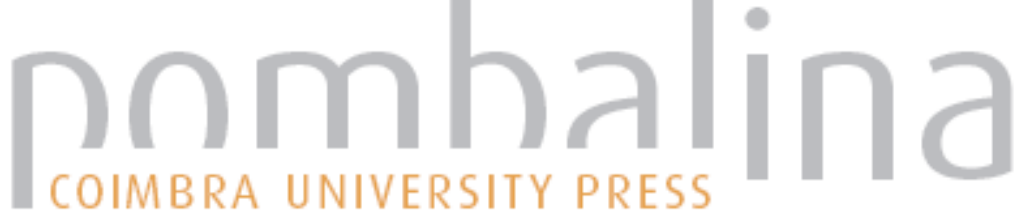

Lutter sans espoir ou se révolter par solidarité: notes sur la pensée d'Albert Camus

Autor(es): Domingues, João

Publicado por: Imprensa da Universidade de Coimbra

URL

persistente: $\quad$ URI:http://hdl.handle.net/10316.2/38689

DOI: $\quad$ DOI:http://dx.doi.org/10.14195/978-989-26-1164-8_12

Accessed : $\quad$ 26-Apr-2023 14:39:36

A navegação consulta e descarregamento dos títulos inseridos nas Bibliotecas Digitais UC Digitalis, UC Pombalina e UC Impactum, pressupõem a aceitação plena e sem reservas dos Termos e Condições de Uso destas Bibliotecas Digitais, disponíveis em https://digitalis.uc.pt/pt-pt/termos.

Conforme exposto nos referidos Termos e Condições de Uso, o descarregamento de títulos de acesso restrito requer uma licença válida de autorização devendo o utilizador aceder ao(s) documento(s) a partir de um endereço de IP da instituição detentora da supramencionada licença.

Ao utilizador é apenas permitido o descarregamento para uso pessoal, pelo que o emprego do(s) título(s) descarregado(s) para outro fim, designadamente comercial, carece de autorização do respetivo autor ou editor da obra.

Na medida em que todas as obras da UC Digitalis se encontram protegidas pelo Código do Direito de Autor e Direitos Conexos e demais legislação aplicável, toda a cópia, parcial ou total, deste documento, nos casos em que é legalmente admitida, deverá conter ou fazer-se acompanhar por este aviso. 
João Domingues

Universidade de Coimbra / Centro de Literatura Portuguesa

\title{
LUTTER SANS ESPOIR OU SE RÉVOLTER PAR SOLIDARITÉ. NOTES SUR LA PENSÉE D'ALBERT CAMUS
}

\author{
«Un rationalisme de l'irrationnel, \\ une philosophie sombre des lumières» \\ (Emmanuel Mounier)
}

Désigner Camus un écrivain existentialiste n'est qu'une tentative, très peu précise d'ailleurs, de situer cet auteur dans son époque, alors que sa pensée révèle de multiples facettes et n'est pas facile à classer. Pour l'intellectuel tout comme pour le citoyen, face à l'agitation du monde de son époque, l'engagement était un devoir inévitable, et la littérature en était une forme par excellence. À cette époque-là, les écrivains, comme les autres hommes, sont emportés par les événements qui se précipitent. Puis, abasourdi par les crimes de toute sorte et par les horreurs de la guerre, Camus, et tant d'autres, hésite entre la désillusion, le désespoir - ou l'absurde - et le redressement de nouvelles valeurs.

Camus se trouve d'abord profondément partagé entre son attachement à la pauvre terre arabe qui l'a vu naître, sèche et poussiéreuse, et une aspiration scintillante que seul le soleil d'Afrique du Nord pourrait avoir illuminée. L'homme d'abord, et puis l'écrivain, se placent donc, dès le début, sous les auspices d'une lumière féconde.

Chacune de ses œuvres exprime tout aussi bien l'engagement de sa pensée que celui de sa personne; ses écrits sont inséparables des événements 
de sa vie, car il ne s'est jamais tenu à l'écart des combats, des échecs ou des convulsions de l'histoire. Et sa pensée dite "ondulatoire", hésitant bien souvent entre le "désespoir solitaire» et la «révolte solidaire», est sûrement le résultat d'expériences multiples et contradictoires, tantôt éprouvées par le corps tantôt ressenties par l'esprit.

En Algérie, dit-il, le don du soleil enrichit de sa lumière le quotidien de ceux qui y vivent. Entre l'espace lumineux de son enfance, partagé, certes, avec la misère algéroise, et les lieux de son ascension à la splendeur littéraire, Camus a certainement connu des moments de découverte de "l'envers nocturne" de la souffrance auquel sa propre santé fragile n'a pas su l'épargner. Et, dès lors, sa vision de la condition humaine fut inéluctablement porteuse de ce double visage de Janus: ombres et lumière ou, selon Camus lui-même, "l'envers et l'endroit» de son monde, et du monde en soi. Entre le soleil invincible omniprésent dans Noces (1939) et le plaisir profond de goûter «'eau et le soleil, les pierres chaudes et la mer» - qui ont même fait que Sisyphe «ne voulut plus retourner dans l'ombre infernale» (Camus, 1942a , 2006: 164) -, il a dû parcourir maints chemins raboteux et traverser beaucoup d'ombres.

Situés pour la plupart en Afrique, les récits et les romans de Camus sont inondés du soleil et de la lumière du ciel africain, tantôt illuminant tantôt écrasant de son intensité la terre et les hommes. En ce sens, notre quête tentera de mettre en relief tout ce que cette lumière apporte à l'expression de sa pensée: recours poétique obsédant dans tout son imaginaire, la présence de la lumière s'élève souvent au rang de métaphore et même de "personnage» omniprésent. Par contre, s'il lui faut montrer l'absence de cette lumière, c'est aux antipodes des ciels lumineux (autant pour les lieux que pour les faits et leur signification ), qu'il a recours, à savoir le ciel sombre d'Amsterdam, ville aux eaux livides, aux terres grises. De la plus innocente clarté de l'aurore (apaisante pour le condamné en prison dans L'Étranger, ou simplement excitante pour le jeune algérois du Premier homme), à la lumière écrasante de ce soleil arabe le jour des funérailles de la mère de Meursault, d'innombrables nuances, d'ombres et de lumière, accompagnent ou expriment l'ébauche d'une pensée "ondulatoire» et font preuve d'un vécu inquiet, toujours en quête de sens. 


\section{Des lumières de la poésie aux feux du combat}

«Si je n'ai pas dit tout le goût que je trouve à la vie, toute l'envie que j'ai de mordre à pleine chair, si je n'ai pas dit que la mort même et la douleur ne faisait qu'exaspérer en moi cette ambition de vivre, alors je n'ai rien dit.»

"Constater l'absurdité de la vie ne peut être une fin, mais seulement un commencement. [...] Ce n'est pas une découverte qui intéresse, mais les conséquences et les règles d'action qu'on en tire.» ${ }^{2}$

"Miracle d'aimer ce qui meurt»3. Immergé dans la beauté du paysage de Tipasa, Camus célèbre, dans le recueil Noces, l'union profonde de l'homme avec la nature. Dans cet espace lumineux où tout semble prendre vie par la lumière, la communication entre l'homme et la nature prend une dimension presque mystique, et la nature devient alors extase: en effet, envahi par «l'odeur des absinthes», entouré par des fleurs, "une mer cuirassée d'argent» et de la "lumière à gros bouillons», "les yeux tentent vainement de saisir autre chose que des gouttes de lumière et de couleurs qui tremblent au bord des cils» ${ }^{4}$. Dans le récit, surgit d'abord un paysage de rêve sous un climat paradisiaque et une atmosphère singulière aux accents lyriques bouleversants, où l'homme se dilue et jouit du bonheur d'exister; et c'est un art de vivre le présent que cette «entente amoureuse de la terre et de l'homme», un bonheur presque parfait qui, dans la joie, s'oppose obstinément à l'absurde. Comme ébloui par la lumière, l'humain se délecte alors dans cette union matricielle, dans un bonheur de rêve poétique.

Tout au long des quatre récits qui composent Noces, l'exaltation de la nature surgit comme un seul flot poétique et rêveur; l'homme s'y trouve épousé et béni par la terre et par la lumière omniprésente. L'intensité lumineuse produit d'ailleurs chez l'homme un sentiment si unique et si fort qu'il comble tous ses désirs:

1 Lettre de Camus à Jean de Maisonseul, apud: Roger Grenier, 1987: 64.

2 Extrait d'une critique de Camus à La Nausée de J. P. Sartre, parue dans Alger-Républicain. Apud: R. Grenier, 1987: 124.

3 Il s'agit d'un vers de la chanson que Camus avait écrite sur «la Maison devant le monde», se rappelant du bonheur qu'il y avait vécu. Apud: Roger Grenier, 1987: 22.

4 A. CAmus, Noces à Tipasa, CEuvres Complètes, éd. Pléiade, p. 55. 
Hors du soleil, des baisers et des parfums sauvages, tout nous paraît futile. [...] Ici, je laisse à d'autres l'ordre et la mesure. C'est le grand libertinage de la nature et de la mer qui m’accapare tout entier. [...] Que d'heures passées à écraser les absinthes, à caresser les ruines, à tenter d'accorder ma respiration aux soupirs tumultueux du monde! [...] j'ouvre mes yeux et mon cour à la grandeur insoutenable de ce ciel gorgé de chaleur. Ce n'est pas si facile de devenir de qu'on est, de retrouver sa mesure profonde. [...] J'apprenais à respirer, je m'intégrais et je m'accomplissais. (Ibidem: 56)

La conquête du bonheur exige, cependant, que l'homme devienne ce qu'il est vraiment, qu'il remonte à son origine, et qu'il refasse son expérience originelle. Pour être en accord avec le monde naturel, il doit se libérer de tout et, redevenu créature originelle, apprendre qu' «il n'y a pas de honte à être heureux». La jouissance pure, cette expérience d'avant le péché, sorte d'hédonisme au-delà de toute morale prôné par Camus, révèle l'homme à l'état pur, l'homme originel

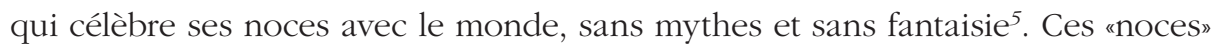
expriment par elles-mêmes l'accord de l'homme avec son destin mortel. Et vivre ainsi c'est accepter de bien jouer son rôle, tel l'acteur qui réussit à faire coïncider ses gestes avec ceux du personnage idéal qu'il incarne. Et dans ces conditions, c'est "un devoir d'être heureux» ${ }^{6}$.

Moins lyrique, surtout quand il décrit la vie de ses habitants dans "L'été à Alger», le récit devient plus sobre, presque austère. Mais, chaque rue débouchant sur la mer, et le soleil inondant de sa lumière chaque recoin de la ville, l'homme y est toujours comblé, et ce même s'il est pauvre. Comblé ne serait-ce que de cette lucidité qui lui permet de voir et de désirer, et de "mesurer ses richesses» au lieu de vivre dans la nostalgie: "ce pays ne promet ni ne fait entrevoir. Il se contente de donner, mais à profusion»”; la vie s'y épanouit alors tout naturellement sous une lumière bienfaisante. La lumière, maîtresse de l'existence, rythme

5 A. CAMus, Le désert, CEuvres Complètes, éd. Pléiade, p. 84: «car les mythes sont [...] des masques ridicules posés sur la passion de vivre».

6 A. CAMUS, "Noces à Tipasa", CEuvres Complètes, éd. Pléiade, p. 60.

7 A. CAMUS, "L'été à Alger", in Essais, éd. Pléiade, p. 67. 
à son gré toute la vie, de l'homme et de la nature ${ }^{8}$. Lumière étrange que celle d'un pays qui, malgré sa misère, nous enchaîne et nous fait dire que «oui, c'est là-bas qu'il nous faut retourner».

Dans «Le désert» cohabitent harmonieusement la beauté et la mort, le bonheur de l'homme et sa propre finitude, à travers l'évocation de paysages et de peintures. En effet, même si la mort existe, tout comme les soirs - heure triste "dans le ciel sans lumière" ${ }^{9}$-, devant la mort le soleil sera toujours là pour nous réchauffer; et quand bien même on serait devant l'envers du monde, il serait encore là $^{10}$. Et "qu'est-ce que le bonheur sinon le simple accord entre un être et l'existence qu'il mène?,"11

Dans les récits qui composent «Noces», sans jamais cesser de proclamer la joie de vivre et la soif de trouver sur cette terre le bonheur, on voit néanmoins apparaître la présence, bien qu'un peu secrète, de la mort qui menace ce bonheur; en fait, "tout ce qui exalte la vie accrô̂t en même temps son absurdité». L'homme ne trouvera-t-il jamais le sens de la vie? Ce sentiment de joie et de lumière serait-il suffisant pour que la vie vaille la peine d'être vécue? Refusant le suicide, plus par instinct que par raisonnement, les yeux rivés sur l'absurde, l'homme pourrait-il alors, lucide et libre, se révolter et vivre ses passions? Vivre de ses passions? Quoi qu'il en soit, le lyrisme de Noces nous prépare déjà à la compréhension de ses autres approches de la vie et de l'homme, marquées par cette profonde inquiétude pour l'être humain. Par ailleurs, on trouve clairement formulé - bien

\footnotetext{
8 Pour les corps, par exemple, c'est la lumière de l'été qui détermine son évolution en couleur, qui passe «du blanc au doré, puis au brun, et pour finir à une couleur tabac qui est la limite extrême de l'effort de transformation dont le corps est capable[...]». En revanche, "quand on est au niveau de l'eau, sur le fond blanc cru de la ville arabe, les corps déroulent une frise cuivrée. Et, à mesure qu'on avance dans le mois d'août et que le soleil grandit, le blanc des maisons se fait plus aveuglant et les peaux prennent une couleur plus sombre. Comment alors ne pas s'identifier à ce dialogue de la pierre et de la chair à la mesure du soleil et des saisons?’ (p. 69). Tout est jeu d'ombre et de lumière, et les silences même de la ville sont différents "selon qu'ils naissent de l'ombre ou de la lumière»: à l'heure où le soleil déborde de tous les coins du ciel, "le silence de midi sur la place du Gouvernement» est bien différent de celui dont on jouit à l'ombre des arbres d'où les Arabes, vendant de la citronnade, font voler l'appel «fraîche, fraîche», qui traverse la place déserte. "Après leur cri, le silence retombe sous le soleilw (p. 70). Le soir enfin, dès que la première étoile se rend visible, arrive aussitôt, dévorante, la nuit; mais "ces courts instants où la journée bascule dans la nuit» sont autant de "promesses de bonheur": une "étrange lumière verte, née du double coquillage du ciel et de la mer» (p. 71), surgit alors réveillant l'homme pour les charmes de la nuit à Alger. (Ibidem: 69 - 71)

9 A. CAMUS, L'envers et l'endroit, CEuvres Complètes, éd. Pléiade, p. 23.

10 «Une pellicule de soleil qui craquerait sous l'ongle, [...] revêt toutes choses d'un éternel sourire». Ibid., p. 48.

11 A. CAMUS, "Le désert", in Essais, éd. Pléiade, p. 85.
} 
que toujours avec un certain lyrisme - le sentiment de l'absurde et de la révolte, et ce malgré toute cette lumière qui invite au bonheur.

L’homme révolté (1951) est déjà le condensé de toutes les révoltes; c'est l'histoire de l'homme qui, refusant le nihilisme, cherche malgré tout un espoir, un soleil qui s'impose sur toute l'humanité et qui puisse, en quelque sorte, la nourrir de sa lumière. Tout individu, maître ou esclave, bourreau ou victime, prenant conscience de soi et voulant se faire respecter pour ce qu'il est, se révolte - car «la conscience vient au jour par la révolte» ${ }^{12}$ - et veut agir au nom de ce bien qu'il «met au-dessus du reste et [...] proclame préférable à tout, même à la vie» (Ibidem: 424). Or, c'est par ce même mouvement de révolte qu'il a «le soupçon qu'il y a une nature humaine [...]», car "pourquoi se révolter s'il n'y a, en soi, rien de permanent à préserver?» (Ibidem: 425). Passionnée et profondément positive $^{13}$, la révolte «révèle ce qui, en l'homme, est toujours à défendre» (Ibidem: 429). Elle est, donc, cette lumière qui permet à l'homme de passer de la notion d'absurdité et de stérilité d'un monde radicalement égoïste, où la souffrance même n'est affaire que d'une seule personne (le malade ou la victime), à une expérience partagée par toute l'humanité: saisi d'abord par l'étrangeté du monde, l'esprit réussit, par la révolte, à reconnaître que cette étrangeté est partagée par tous les humains qui, sous un même soleil, éprouvent le même mal, comme s'il était devenu "peste collective ${ }^{14}$ qu'il faudrait, par conséquent, combattre ensemble. Par le «je me révolte, donc nous sommes», Camus - à l'instar du docteur Rieux dans La Peste (1947) - aurait alors entrevu le seul vrai combat qu'il fallait mener, la seule issue possible pour l'homme face à ce monde à refaire.

\section{Lumières aveuglantes et inquiétudes}

Dans L'Étranger (1942), comme dans Le Mythe de Sisyphe (1942), l'homme est jugé pour ce qu'il est dans son "être là», autrement dit, pour son existence

\footnotetext{
12 A. CAMUS, L'Homme révolté, éd. Pléiade, p. 424.

13 Contrairement au ressentiment, qui est, à son tour, un sentiment profondément négatif, selon Camus.

14 Ibidem, p. 432: "reconnaître qu'il partage cette étrangeté avec tous les hommes et que la réalité humaine, dans sa totalité, souffre de cette distance par rapport à soi et au monde. Le mal qu'éprouvait un seul homme devient peste collective».
} 
qui hélas ne trouve pas sa raison d'être. Et, en effet, le soleil, bien qu'il règne sur toutes choses, écrase de tout son poids les hommes et la nature, plus qu'il n'illumine. Et si «noces» il y a entre l'homme et toute la nature, elles seraient devenues «infernales»! L'Étranger est plutôt une tragédie de la lumière, car ce soleil, s'il recouvre la laideur de la misère à Alger, il met à nu la misère humaine la plus grande, à savoir l'absurdité de son existence.

Dans ce roman, la lumière règne, mais elle n'éclaire pas; au contraire, elle est la déchirure même qui germe au plus profond du protagoniste. Depuis les funérailles de sa mère, et jusqu'au moment ou il attend d'être conduit à l'échafaud, rien n'exprime mieux chez Meursault cette course insensée de l'homme vers la rencontre inexorable de sa destinée que cette chaleur et cet excès de lumière d'un ciel trop bleu ou trop blanc. Rien de plus aveuglant que ce «soleil débordant qui faisait tressaillir le paysage [...] inhumain et déprimant» (Camus, 1942², 2005: 27), que ce jour «tout plein de soleil» qui le frappe «comme une gifle» (Ibidem: 77), que ce ciel qui s'ouvre «sur toute son étendue pour laisser pleuvoir du feu» (Ibidem: 94). Dans un scénario méticuleusement assemblé, la lumière, tantôt bénéfique tantôt meurtrière, rôde ou plane au-dessus du héros, dans une ambiance laborieusement façonnée et le domine tout entier, jusqu'à perdre haleine.

Entre l'inquiétude - obnubilée sous la cape d'une espèce d'indifférence d'avoir à vivre sans rien comprendre, et la révolte que l'on perçoit derrière la constatation de l'absurde, la lumière, l'excès de lumière, est sans doute la manifestation de tout ce qu'il nous faut vivre mais qui dépasse notre entendement. Et, dès lors, un choix s'impose: soit on refuse et on coupe court par le suicide, ce qui n'est pas une solution pour Camus ${ }^{15}$, soit on accepte humblement notre "Condition limitée» (Ibidem: 417). Curieusement Meursault, le protagoniste, n’incarne apparemment ni l'un ni l'autre; il est plutôt comme un arrêt de jugement, un être séduit par «la tendre indifférence du monde» (Camus, 1942 , 2005: 186). En ce sens, il nous montre que, même si on est un jour victime de la justice toute apparente et ô combien déraisonnable et injuste, voire même criminelle -, on pourra néanmoins toujours se réjouir d'avoir connu, d'avoir aimé, d'avoir

15 "On ne résout pas un problème en l'évitant", a-t-il répondu un jour à Pascal Pia. Dans cet autre passage - parmi d'autres encore - il refuse péremptoirement le suicide: "La conclusion dernière du raisonnement absurde est, en effet, le rejet du suicide et le maintien de cette confrontation désespérée entre l'interrogation humaine et le silence du monde" A. CAMUS, "L'Homme révolté", in Essais, éd. Pléiade, p. 415. 
été quelqu'un, pendant un certain temps, peut-être même heureux, quelque part ici entre "la mer, le sable et le soleil» (Ibidem: 91). Cette lumière d'Afrique, comme cette «pluie aveuglante qui tombait du ciel» (Ibidem: 91) en permanence, sont aussi bien l'union la plus profonde entre ces trois éléments que la preuve de tout ce qui dépasse notre regard et qui, à notre insu, commande en quelque sorte, nous commande peut-être, et ne peut que nous éblouir! Si la terre et tout ce qui l'habite est le fruit du regard des dieux, la lumière en est certainement la demeure. Coincé entre ciel et terre, Meursault a souvent «envie de fuir le soleil [...], envie de retrouver l'ombre et le repos» (Ibidem: 92), où qu'ils se trouvent.

Il serait tentant de percevoir, derrière ce héros hanté par le soleil qui «se brisait en morceaux sur le sable et sur la mer» (Ibidem: 89), en des moments où il n'y avait "plus eu que le soleil et le silence» (Ibidem: 89), et en quête d'un sens existentiel, l'auteur, lui-même jeune algérois comme son héros, faisant de son acte d'écriture une catharsis personnelle. Camus, tout comme son héros, se situe, à la fin de ce parcours, aux frontières qui limitent toute existence: Meursault trouve à la fin "la tendre indifférence du monde»(Ibidem: 186); mais, Camus, peut-il en dire autant? L'écriture de ce roman le réconcilie peut-être quelque peu avec ses origines matricielles - sa jeunesse pauvre mais lumineuse à Alger - et avec sa propre mère; et il s'en délivre. Mais se sent-il vraiment libéré? En fait, alors que Meursault, "pour que tout soit consommé» (Ibidem: 186), n'a qu'à attendre le jour de l'exécution, Camus, lui, l'homme et le penseur-écrivain, sait que d'autres jours et d'autres ciels se lèveront encore; avec plus ou moins de lumière.

\section{Du tragique et de l'absurde}

«L'absurde naît de la confrontation de l'appel humain avec le silence déraisonnable du monde ${ }^{16}$

16 A. CAMUS, Le Mythe de Sisyphe (1942a, 2006: 46). Cette même pensée l'auteur l'avait déjà exprimée, de manière un peu plus développée, quelques pages auparavant: "Ce monde en lui-même n'est pas raisonnable, c'est tout ce qu'on en peut dire. Mais ce qui est absurde, c'est la confrontation de cet irrationnel et de ce désir éperdu de clarté dont l'appel résonne au plus profond de l'homme. L'absurde dépend autant de l'homme que du monde» (Ibidem: 39). 
Dans Caligula (1941), il n'est pas question d'ombres ou de lumière naturelles, mais plutôt d'une espèce de lumière intérieure, brûlante et aveuglante à la fois, jusqu'à la folie; elle est la sour jumelle des ombres de la déraison et du crime. A la lumière d'une clairvoyance dérangeante, l'empereur nous fait voir d'abord une vérité simple, qui ne relève que de la constatation, bien que très lourde à porter, ćest-à-dire que "les hommes meurent et ne sont pas heureux" ${ }^{17}$; par conséquent, Caligula aura besoin de «la lune», autrement dit, de l'impossible. En effet, quand on découvre que l'on est mortel et malheureux, plus rien ne vaut et rien ne va plus. Impossible de concevoir que ce monde soit en ordre, car plus rien n'a de sens: ni les liens de sang, ni les affections, rien. Or, face à ce mal-être essentiel, accorder de l'importance au pouvoir ou à l'argent serait alors le comble de la dérision. Par conséquent, Caligula n'est ni un criminel ni un monstre quand il dit que "tout cela manque de sang»; il est plutôt une âme lucide, en quête de sens pour soi, à la recherche de la solution, sans contraintes, qu'elle soit l'envers du monde - déshéritant les enfants des propriétaires ou tuant arbitrairement n’importe qui - ou tout simplement le désir de l'impossible; désirer la lune, cette ombre qui revient cycliquement sur la voute du ciel, s'offrant à nous sans que jamais personne n'ait été capable de la posséder. L'envers du monde, ou le monde à l'envers, la lune à la place du soleil, voilà ce qui semblerait plus juste, plus clair, plus conséquent, et peut-être moins déraisonnable que l'ordre de ce monde où une guerre tue mille fois plus de gens que tous les caprices sanguinaires de Caligula; et pourtant c'est lui qu'on surnomme «lempereur fou».

Tout en traduisant un manque absolu de lumière, Le Malentendu (1944) met en relief l'absence d'ordre dans ce monde ainsi que son manque de sens. En "ce pays d'ombre»18, le seul ordre possible est "celui où personne n'est jamais reconnu" (Ibidem: 178). En effet, en absence de lumière, pas même le sang familial n'est reconnaissable. Mère et fils étant devenus, par l'absence de lumière, assassin et victime, plus rien ne pourra les éclairer: ils restent des êtres de la nuit pure. Plus tard, ils se rejoindront, certes, mais hélas déjà sous «cette terre épaisse, privée de lumières» (Ibidem: 178), dans l'ombre de la mort; et ils ne se reconnaîtront

17 A. CAMUS, Caligula, éd. Pléiade, p. 16.

18 A. CAMUS, Le Malentendu, éd. Pléiade, p. 117. 
plus. C'est l'expression du vrai tragique, car rien ni personne ne pourra plus jamais apporter de solution à ce crime.

Mais c'est avec La Chute (1956) que nous parvenons aux antipodes de la lumière, là où siègent les ombres du nord. Cependant, entre Alger et Amsterdam, un lien étroit se tisse: celui du fil ininterrompu de l'humain, tendu entre les forces de la lumière et celles des ombres, entre le désir de voir et de comprendre - qui finit par aveugler tout un chacun - et le propos de se soumettre à une pénitence purificatrice de l'âme, pénitente ou révoltée, mais toujours nécessairement déchue en ce monde. L'action se déroule dans un lieu bas et sombre. Clamence, le juge-pénitent, est un être déchu qui vit dans ce lieu plat, au-dessous du niveau de la mer, enfin dans un enfer appelé Amsterdam où la lumière est la grande absente. Car l'envers de la vie n'est pas la nuit d'Afrique; il s'apparente plutôt au ciel d'Amsterdam sous lequel, pour se réjouir, on n'a que «le genièvre, la seule lueur dans ces ténèbres»" ${ }^{19}$. De même, la vie de Clamence est l'envers d'une vie d'homme; les historiens l'auront bien définie un jour, avec ô combien de clarté et de précision, quand ils diront que l'homme moderne était celui qui «forniquait et lisait des journaux». Comme le dit Clamence lui-même, "après cette forte définition, le sujet sera, si j'ose dire, épuisé» (Ibidem: 1479). Mais il faudra aussi noter, pour l'histoire de la société européenne, qu'il est une autre partie de cet envers, aux allures un peu différentes, c'est vrai, mais nullement moins éloquente, à savoir: les «soixante-quinze mille juifs déportés ou assassinés»; la vieille femme courtoisement invitée par un officier allemand à "choisir celui de ses deux fils qui serait fusillé»; le pacifiste qui avait écrit sur le seuil de sa maison «d'où que vous veniez, entrez et soyez les bienvenus» (Ibidem:1481), et que, par conséquent, des miliciens «étripèrent» en pénétrant chez lui.

L'envers du monde est aussi ce lieu "coincé dans un petit espace de maisons et d'eaux, cerné par des brumes de terres froides, et la mer fumante comme une lessive» (Ibidem:1484); et l'envers de l'humain «ces messieurs-ci [...] qui vivent du travail de ces dames-là» ( Ibidem: 1479) et "ces dames, derrière ces vitrines» (Ibidem: 1483). L'envers 'chaud' à Amsterdam, ou l'enfer beau à Red Light Street!

L'envers de l'humain est long à parcourir; il nous semble, d'ailleurs, qu'il ne cessera jamais d'exister, voire de grandir. Il serait tentant ici de pérorer longuement

19 A. CAMUS, La Chute, éd. Pléiade, p. 1481. 
sur l'histoire sombre de cet industriel qui, infidèle, et ne supportant pas d'avoir une femme parfaite, décida de couper court et de la tuer; on laisse aussi de côté l'affaire de la concierge qui, lors des funérailles de son mari, «s'était ruinée en crucifix, en beau chêne, et en poignées d'argent, pour mieux jouir de son émotion", et qui "s'est collée, un mois plus tard, avec un faraud à belle voix" (Ibidem: 1493-94). Prenons juste la vie de notre juge-pénitent Clamence, vénéré en tant qu'avocat des bonnes causes, défendant la veuve et l'orphelin, et qui en vérité crève de vanité. Il se croit le plus intelligent et le plus vertueux; simple fils d'officier, il se prend pour un fils de roi (Ibidem: 1490); et au moment de pratiquer la vertu, d'appeler au secours pour la jeune femme inconnue - qu'il venait de croiser sur le Pont Royal, après une belle soirée de plaisir - qui se noyait dans la Seine, il ne bougea pas, et se trouva même de bonnes excuses pour ne pas bouger (Ibidem: 1511). «'ai compris», dira-t-il plus tard, "que la modestie m'aidait à briller, l'humilité à vaincre et la vertu à opprimer» (Ibidem: 1518). L'envers de l'homme, l'envers des valeurs qu'on aurait cru sûres et vraiment humaines. La face des vertus n'est donc jamais dépourvue de son revers, comme cet avocat des bonnes causes qui n'était qu'un «dernier hommage que le vice rend à la vertu». Chez Camus, la pensée moraliste remplit souvent tout le discours d'une ironie sombre et amère, comme une obsession.

Aux antipodes des beaux ciels d'Alger, de la terre chaude et de la mer scintillante, l'île de Marken est "le plus beau des paysages négatifs»:

[...] ce tas de cendres qu'on appelle ici une dune, la digue grise [...], la grève livide à mes pieds et, devant nous, la mer couleur de lessive faible, le vaste ciel où se reflètent les eaux blêmes. Un enfer mou, vraiment! [...] aucun éclat, l'espace est incolore, la vie morte. N'est-ce pas l'effacement universel, le néant sensible aux yeux? (Ibidem: 1512)

Immergé dans cet environnement purement négatif, Clamence devient alors une figure emblématique «de la débauche et du malconfort». Clamence prend-il sa propre vie amoureuse pour sujet? Il se voit comme un abîme d'égoïsme, car il n'aime que lui. Il se fait aimer pour le plaisir; et quand il décide d'arrêter le jeu, c'est-à-dire hors de tout jeu, en dehors du désir, les femmes l'ennuient. Arrivé enfin à la vérité, le malconfort l'agace, car "la vérité est assommante» 
(Ibidem: 1527), hélas! Nourrissant, depuis longtemps, le projet d'aller à la rencontre de la vérité, Clamence finit par s'engouffrer dans les brumes du nord. La débauche devint une sorte d'avatar de l'immortalité tant désirée: l'alcool, les femmes, la nuit tiennent lieu de libérateurs, car il n'en découle aucune obligation; les lieux où elle s'exerce semblent séparés du monde; "elle est une jungle, sans avenir ni passé, sans promesse surtout, ni sanction...", la libération, cette absence de limites qui nous rend immortels, quoique pour quelques instants seulement. Mais les corps ne sont pas faits pour l'immortalité, même pas pour cette immortalité temporaire qu'est la débauche; car bientôt le désir s'amollit, le foie se plaint, les forces fléchissent et le corps cède; quel avantage, alors, à vivre dans cette «sorte de brouillard?» (Ibidem: 1530) La guérison se révèle éphémère, et le cri de la noyée de la Seine ne cesse de le hanter, l'attendant «sur les mers et les fleuves, partout enfin où se trouverait l'eau amère de [son] baptême» (Ibidem: 1531): l'eau, toujours les eaux, scintillantes et maternelles de la Méditerranée, ou alors sombres, lourdes, inquiétantes, de Paris sous un brouillard hivernal, ou de ce «bénitier immense» (Ibidem: 1531) des rivages d'Amsterdam.

La vie glorieuse du Juge s'achevant, «il fallait se soumettre et reconnaître sa culpabilité» et faire pénitence. "Il fallait vivre dans le malconfort» (Ibidem: 1531). Quelle ironie amère, mais combien de lucidité si l'on tient compte de l'existence humaine au cours du xxème siècle. Quelle clairvoyance, seule digne d'un juge, et quelle proximité, à la fois, du pénitent face à la réalité éprouvée par toute cette génération de l'après-guerre dont les écrits sont des miroirs qui crachent toutes ces vérités aux yeux de l'hypothétique lecteur; il faudrait être né malvoyant pour ne pas se sentir étourdi et aveuglé par la simple vision de tant d'éclats mortels. Il faudrait être né insensible pour ne pas endurer tout le poids que l'histoire récente a mis sur nos épaules, jusqu'à nous tordre le dos; car ce monde est devenu une cellule "pas assez haute pour qu'on s'y tînt debout, [...], pas assez large pour qu'on pût s'y coucher» (Ibidem: 1531). C'est pour cela que nous, les condamnés, nous sommes obligés de vivre «en diagonale».

Dans ce panorama existentiel, la morale n'a pas de place, la morale n'est pas, et seul le moraliste a raison; selon ce regard tout voltairien, "Dieu n'est pas nécessaire pour créer la culpabilité, pas plus que pour punir. Car nos semblables y suffisent, aidés par nous-mêmes»; et, à en juger par tous leurs chefs-d'œuvre 
de crimes perpétrés, ils y suffisent fort bien. Pas besoin de jugement dernier non plus, car «il a lieu tous les jours». (Ibidem: 1532)

Avouons enfin, avec Clamence, que «'ordre du monde est ambigu»; son Sauveur était, selon toute apparence, coupable lui-même, certes pas d'avoir tué quelqu'un, mais d'avoir laissé mourir tous ces innocents: partout devrait le poursuivre "la voix de Rachel, gémissant sur ses petits et refusant toute consolation". Partout "la plainte s'élevait dans la nuit, Rachel appelait ses enfants tués pour lui, et il était vivant!» (Ibidem: 1533) La nuit de l'infanticide en Israël, la nuit de la noyade à Paris, la nuit de débauche et de brouillard à Amsterdam, autant de nuits de Gethsémani, affreusement douloureuses, parce que dépourvues de sens, de but et de cause, où en finir serait le moindre mal, voire un soulagement, car le plus dur c'est encore de continuer, de tenir, de rester, de résister, d'être là. Voilà ce que nous apprend Clamence le juge-pénitent, Clamence le prophète du malheur "réfugié dans un désert de pierres, de brumes et d'eaux pourries, [...] le doigt levé vers un ciel bas». (Ibidem: 1535)

Peut-on alors affirmer que, puisqu'il n'y a pas de sens pour la morale, rien n'a de sens? Certainement pas; car une simple histoire aussi fantaisiste soit-elle, a un sens; plus ou moins vraie, elle aura un sens; même l'histoire purement fausse a un sens, le sens d'une fausseté. L'immoraliste a donc tort, le nihiliste aussi ${ }^{20}$, car, si éloignée qu'elle soit de nous, il doit y avoir une issue, une solution, une vérité. Mais «la vérité, comme la lumière, elle aveugle. Le mensonge, au contraire, est un beau crépuscule» ${ }^{21}$; ce crépuscule, propre de l'homme, est une espèce de mediocritas, à mi-chemin entre l'exubérance de la lumière aveuglante du désir de bonheur et d'éternité, et le brouillard lourd et triste de nos limites.

Après avoir décidé de venir travailler dans cette "capitale d'eaux et de brumes» (Ibidem: 1547), et étant devenu receleur d'un tableau volé, cherchant sans cesse quelqu'un qui le dénonce, le condamne et l'emprisonne - lui donnant ainsi la possibilité de faire pénitence -, Clamence découvre qu'il est trop tard; la jeune

20 Le texte "Au-delà du nihilisme" commence d'ailleurs de manière très explicite: "Il y a donc, pour l'homme, une action et une pensée possibles au niveau moyen qui est le sien". A. CAMUS, Essais, éd. Pléiade, p. 705.

De même pour ce qui est de la philosophie de l'absurde, car, ne dit-il pas: “... la position absurde, en acte, est inimaginable. Elle est inimaginable aussi dans son expression. Toute philosophie de la non-signification vit sur une contradiction du fait même qu'elle s'exprime”. A. CAMUS, "L'Homme révolté", in Essais, éd. Pléiade, p. 418.

21 A. CAMUS, La Chute, éd. Pléiade, p. 1537. 
fille ne se jettera pas une seconde fois dans les eaux de la Seine, pour qu'il puisse "s'exécuter». "Il est trop tard [...], il sera toujours trop tard» (Ibidem: 1551); car, comme les eaux qui ne passent jamais deux fois sous le même pont, dans la tragi-comédie de la vie il n'y a pas de répétition, pas d'entraînement possible: sans jamais pouvoir recourir aux coulisses, on est toujours sur scène et on ne peut jouer notre rôle qu'une fois.

Des lumières africaines jusqu'à Paris et Amsterdam, le chemin est aveuglant et long, comme une chute dans un abîme infini où tombera tout être humain dépourvu d'espoir; quel dommage que ce voyage vraiment initiatique ne se fasse pas dans le sens contraire, à savoir non de l'endroit à l'envers, mais bien de l'envers à l'endroit! Car, pour l'homme, c'est peut-être le seul revers important; en tout cas, le seul qui compte vraiment, et le désespère. En effet, au lieu de faire le «saut» vers la transcendance - ou vers Dieu, comme Pascal -, Camus propose, lui, "le pari déchirant et merveilleux de l'absurde. Le corps, la tendresse, la création, l'action, la noblesse humaine, reprendront alors leur place dans ce monde insensé. L'homme y retrouvera enfin le vin de l'absurde et le pain de l'indifférence dont il nourrit sa grandeur»(A. Camus, 1942a, 2006: 77).

\section{4. Étincelles solidaires}

Quelques étincelles, oui, mais sans espoir; car - Le Mythe de Sisyphe et L'Homme révolté nous le montrent bien - parmi les maux de l'Humanité, l'espoir est le plus terrible de tous, puisqu'il équivaut à la résignation. Or vivre c'est se révolter, c'est lutter toujours sans jamais se résigner ni du sort ni de la condition qui nous assiègent, qu'elles s'appellent injustice, misère ou guerre. Voilà comment on pourrait résumer la pensée qu'exprime Camus dans La Peste (1947). C'est toujours en termes d'ombres et de lumière qu'il s'exprime, mais elles y sont d'un autre ordre. En effet, les ombres y sont protectrices alors que la lumière éclaire la démarche humaine; le parcours qui s'y ébauche va de l'incompréhension et du désespoir à la découverte, par la révolte, de la fraternité qu'a fait naître, parfois dans la souffrance la plus aveuglante, la participation et la solidarité. Ombres et lumière laissent entrevoir ici un monde moral qui, tout compte fait, nous parvient et se construit à partir des valeurs les plus simples qui s'imposent à mesure que 
l'action se substitue au sentiment stérile du désespoir. Entre ombres et lumière, l'homme comprend - ou tout du moins accepte - que le mal, la souffrance et la mort, ne sont plus une tragédie absurde quand on a décidé d'avoir le courage de vivre "les yeux ouverts pour la lumière comme pour la mort» (Camus, 1942a, 2006: 76). Situé à Oran, le récit de La Peste est, selon l'auteur, l'expression romanesque du positif. Mais à Oran, outre la lumière, il y a la peste et beaucoup d'ombres! Il y figure surtout l'affrontement entre l'homme et l'absurde de sa propre existence; le premier devrait en sortir vainqueur. Dans cette chronique, le docteur Rieux, narrateur avoué à la fin du récit, veut simplement prouver qu'il y a chez l'homme "plus de choses à admirer que de choses à mépriser». Le comparant à L' Étranger, Camus observe que «La Peste marque [...] le passage d'une attitude de révolte solitaire à la reconnaissance d'une communauté dont il faut partager les luttes». Et il ajoute que "s'il y a évolution de L'Étranger à La Peste, elle s'est faite dans le sens de la solidarité et de la participation»²2.

À Oran, chaque soir, à la tombée de la nuit, l'ombre s'épaissit et les rues s'animent; la nuit venue, les cris des bateaux se mêlent à la rumeur qui monte de la mer et de la foule à "cette heure que Rieux connaissait bien et aimait autrefois» ${ }^{23}$; mais il la trouve maintenant "oppressante à cause de tout ce qu'il savait» (Ibidem: 1265). Dans un monde d'hésitation à l'endroit des malades qui l'attendaient, «il s'attardait à regarder la rue sombre et le ciel noir» (Ibidem: 1266). Face à l'inéluctable constatation de l'épidémie, le docteur se taisait et regardait la dépêche du préfet qui mandait: «Déclarez l'état de peste. Fermez la ville» (Ibidem: 1269). Est-ce l'arrêt de la vie? Est-ce un arrêt de mort? Ceux qui «avaient jusque-là continué de masquer leur inquiétude sous des plaisanteries, semblaient dans les rues plus abattus et plus silencieux" (Ibidem: 1268). Et pourtant, "de beaux bleus ciels" continuaient de déborder de lumière et «tout dans la saison invitait à la sérénité» (Ibidem: 1268). Les vies se consomment, les temps passent, seul le soleil demeure dans sa fixité presque éternelle.

Si la peste avance et Oran se referme sur elle-même, tel un îlot entouré de silence et d'enfer, la ville subsiste "sous un beau ciel» (Ibidem: 1284) donnant l'impression "trompeuse d'une cité en fête dont on eut arrêté la circulation et

\footnotetext{
22 A. CAMUS, Lettre à Roland Barthes, 11 juillet 1955.

23 A. CAMUS, La Peste, éd. Pléiade, p. 1265.
} 
fermé les magasins pour permettre le déroulement d'une manifestation publique, et dont les habitants eussent envahi les rues pour participer aux réjouissances» (Ibidem: 1284). Solennelle indifférence d'une nature aussi pure qu'intouchable, à l'égard des faiblesses d'une humanité fragile et en putréfaction, qu'elle soit charnelle ou morale. Mais cette nature se nourrit-elle vraiment de l'indifférence? Elle qui, devançant même le sermon apocalyptique du père Paneloux, avait, depuis la veille, présenté un ciel assombri d'où «la pluie tombait à verse» (Ibidem: 1296); comme si la nature, contristée et solidaire, avait, à juste titre, été la première à manquer de lumière et à se sentir humiliée par ce sermon écrasant: «méditez cela et tombez à genoux», disait-il; et aussitôt "la pluie redoublait» (Ibidem: 1296). Moment étrange aussi que celui où l'on avoue que la peste est un fléau envoyé du ciel, alors que ce même ciel, qui normalement «débordait de lumière», devient incontinent sombre, comme pour annoncer aux humains qu'ils auront, dès lors, à souffrir "pour longtemps dans les ténèbres de la peste» (Ibidem: 1297).

Après ce prêche, c'est encore la nature, plus précisément la renaissance de la lumière, qui fera penser que cela ne sera pas la fin ${ }^{24}$. Pour le moment, règne "la misère» (Ibidem: 1324); mais c'est à elle que revient le don d'apprendre à l'homme la voie de "la compréhension» (Ibidem: 1325), comme Tarrou avait fini par l'admettre, car c'est elle qui rendra possible le premier pas vers «la lumière». Ainsi, des hommes comme Tarrou se mirent aussitôt au travail pour le bien commun; et, pendant que, "tous les soirs, des commentaires apitoyés ou admiratifs s'abattaient sur la cité désormais solitaire» (Ibidem: 1331), cette "compréhension» les rendaient, eux qui étaient sur place, solidaires: «aimer ou mourir ensemble, il n'y a pas d'autre ressource».

À l'apogée de la peste, le vent dessèche et brûle les rues à longueur de journée, et répand partout la maladie. Le soir, "plongée dans la nuit complète, la ville était de pierre» (Ibidem: 1359): pas un mouvement, pas un bruit, que des

24 Dans la cathédrale, Paneloux finissait par quelques mots d'espoir et d'apaisement et "audehors, la pluie avait cessé. Un ciel mêlé d'eau et de soleil déversait sur la place une lumière plus jeune» (Ibidem: 1299): pour annoncer au monde que, lui aussi, il doit renaître un jour? Peut-être; cependant il fallait encore subir la peste, brûlante comme l'été qui, au lendemain du prêche "éclata d'un seul coup dans le ciel» (Ibidem: 1310), accompagné d'un "grand vent brûlant» qui dessécha tout. Comme la peste, "des flots ininterrompus de chaleur et de lumière inondèrent la ville», et "il semblait qu'il n'était pas un point de la ville qui ne fût placé dans la réverbération la plus aveuglante». Comme la peste, qui était devenue l'affaire de tous, "le soleil poursuivait nos concitoyens dans tous les coins" (Ibidem: 1310). 
cubes massifs inertes et quelques statues au visage de pierre ou de fer. Aussi, "Sous des ciels de lune» ou "sous un ciel toujours épais», ces faux visages trônaient dans les carrefours comme dans une «nécropole où la peste, la pierre et la nuit auraient fait taire enfin toute voix» (Ibidem: 1359). Cette nuit n'était pas seulement physique; elle "était aussi dans tous les coeurs" (Ibidem). De fait, puisque les bienfaits de la lumière ne sont jamais tant appréciés que par ceux qui ont souffert des ombres de la nuit, la peste a fait que, "quoique personne ne fût jamais avec personne» (Ibidem: 1378), en la subissant, les hommes ont appris qu'ils «ne pouvaient pas se passer des hommes» (Ibidem: 1376); ils venaient d'apprendre la solidarité. Cette valeur nouvelle, ou nouvellement apprise, fera que, face au dérisoire humain ${ }^{25}$, et face au scandale qu'est la souffrance et la mort horrible d'innocents $^{26}$, deux choix sont possibles mais un seul est humain, celui qu'a adopté Rambert: "partager le malheur des hommes" et ce même s'il sait qu'il n'aura "plus jamais de temps pour le bonheur» (Ibidem: 1389). Ce "quelque chose qui nous réunit au-delà des blasphèmes et des prières, cela seul est important» (Ibidem: 1397). Et voici que la scène la plus touchante de tout le récit se termine par une union symbolique par excellence: en effet, après avoir assisté à la mort de l'enfant, le docteur Rieux prend la main du père Paneloux et lui dit: "Vous voyez [...] Dieu lui-même ne peut maintenant nous séparer» (Ibidem: 1398); et il prend pour témoin de cette union purement humaine et purement solidaire, la pureté même qu'est «le ciel bleu du matin" d'Oran ${ }^{27}$. Ce même ciel conduit Tarrou

25 Le dérisoire est mis en relief, par exemple, par la mort de l'acteur en scène tel «un histrion désarticulé».

26 Comme cet enfant que la peste transforma, en quarante-huit heures, en "crucifié grotesque» (Ibidem: 1394).

27 La lumière est, tout au long du récit, l'élément naturel le plus présent et le plus compatissant; comme ce jour de La Toussaint où personne ne voulait plus penser aux morts, car "on y pensait déjà trop, justement" (Ibidem: 1411), et, à l'unisson, de gros nuages qui couvraient d'ombres les maisons se dispersaient laissant retomber "la lumière froide et dorée du ciel de novembre» (Ibidem: 1411). Au stade, devenu lieu de quarantaine, il y avait parmi les occupants une certaine méfiance et, en effet, "une sorte de méfiance [...] tombait du ciel gris, et pourtant lumineux, sur le camp rouge" (Ibidem: 1416). Quand Othon, le juge, dit qu'il espère que son fils n'a pas "trop souffert" - alors que l'enfant avait, en fait, subi d'horribles douleurs comparables aux souffrances du crucifié - le soleil lui-même a honte du paradoxe; ainsi «le soleil baissait à l'horizon et, entre deux nuages» - comme par pitié -, "ses rayons entraient [...] dorant leurs visages» (Ibidem: 1417). À la fin de la journée, fatigués d'être là, lassés de ne rien faire, ces hommes en quarantaine se réduisent au son de cuillères et d'assiettes; un crépuscule maternel les couvre alors et, "dans la paix du soir», "une lumière douce et fraîche baignait le camp" (Ibidem: 1417). Fin novembre, malgré les dégâts de l'épidémie, le ciel était nettoyé et les rues luisantes car tous les matins le soleil répandait «une lumière étincelante» (Ibidem: 1418), glacée d'abord, mais qui devenait tiède et accueillante le soir. Aussi, chaque fois que Rieux sortait pour rendre visite à son malade le plus âgé, «le soleil luisait doucement au-dessus des maisons du vieux quartier» (Ibidem: 1418). 
à une espèce de conversion-confession, et fait qu'il choisit le chemin qu'il lui fallait prendre pour arriver à la paix, c'est-à-dire la voie même de «la sympathie» (Ibidem: 1427).

Quant à M. Othon, c'est par un matin d'hiver que le juge d'instruction, à peine sorti de quarantaine, demande au docteur Rieux la permission de devenir volontaire dans l'administration de ce même camp. "Dans ces yeux durs et plats une douceur s’installât soudain», remarque le narrateur, «ils étaient devenus plus brumeux, ils avaient perdu leur pureté de métal» (Ibidem: 1431), comme si, par miracle, la peste avait pu transformer, chez lui, la froideur de la loi en chaleur de solidarité. Aussi Rieux reçoit-il, très calmement au matin, la nouvelle de la mort de sa femme: le télégramme à la main, il avoue à sa mère «que c'était quand-même difficile» (Ibidem: 1460), mais en même temps «il contemplait obstinément, par la fenêtre, un matin magnifique qui se levait sur le port» (Ibidem: 1460); aurait-il enfin compris ce qu'est la vie, c'est-à-dire avoir connu la peste et l'amitié et l'amour et la tendresse ... et s'en souvenir? "Mais si c'était cela gagner la partie, qu'il devait être dur de vivre seulement avec ce qu'on sait et ce dont on se souvient, et privé de ce que l'on espère» (Ibidem: 1459).

Quand «à l'aube d'une belle matinée de février» (Ibidem: 1461) les portes de la ville s'ouvrirent enfin, il y eut des heures de joie. "Des réjouissances étaient organisées pour la journée et pour la nuit» (Ibidem: 1461); et même le soleil, solidaire, "déversait sur la ville des flots ininterrompus d'une lumière immobile» (Ibidem: 1463). Enfin, «tous criaient ou riaient [et] l'égalité que la présence de la mort n'avait pas réalisée en fait, la joie de la délivrance l'établissait, au moins pour quelques heures» (Ibidem: 1464). Mais tous n'étaient pas heureux; parmi ceux qui arrivaient, certains n'avaient pas retrouvé l'être qu'ils attendaient; le bonheur même était pétri d'injustice. En revanche, tous, sans exception, avaient souffert la douleur de l'exil et le désir de réunion; or "quand au sens que pouvaient avoir cet exil et ce désir de réunion, Rieux n'en savait rien» (Ibidem: 1466), et nous n'en savons pas plus, sinon que l'humain se sent ici «naturellement» en exil. Aussi Rieux pense-t-il "qu'il n'est pas important que ces choses aient un sens ou non, mais qu'il faut voir seulement ce qui est répondu à l'espoir des hommes» (Ibidem: 1466). Car nous savons que des gens peuvent parfois être heureux; nous savons aussi qu'il y a «une chose que l'on peut désirer toujours et obtenir quelquefois» (Ibidem: 1467): la tendresse humaine; il nous semble juste aussi «que de temps en 
temps au moins, la joie [vienne] récompenser ceux qui se suffisent de l'homme et de son pauvre et terrible amour» (Ibidem: 1467). Sous «le grand ciel froid» qui scintillait au-dessus des maisons, par une nuit ressemblant à bien d'autres, Rieux avait décidé de rédiger ce récit en souvenir de l’injustice et de la violence faites aux pestiférés, et ainsi nous livrer une vérité qui nous fut révélée par la peste. Sans pour autant justifier l'être humain, elle lui rend néanmoins justice, tant il est vrai "qu'il y a dans les hommes plus de choses à admirer que de choses à mépriser» (Ibidem: 1473).

Dans La Peste, révolte collective contre un mal qui touche tout le monde, la lumière est la première à se déclarer la sour de ceux qui souffrent. À Oran, la lumière est la compagne aimée, toujours attendue pour aider à l'éradication de la peste. Chaque jour, dès qu'elle arrive, la lumière crée comme une tension et l'intensité intérieure qui en résulte devient appel à la solidarité devant le sort funeste de l'autre. La lumière est lucidité et cécité, joie et douleur, espoir et révolte, qui dit toujours oui, oui à la vie, jusque dans la mort. Sous cette lumière, tantôt frappant fort, tantôt touchant juste, la révolte n'est plus négation, mais plutôt acceptation d'un destin que l'on construit au jour le jour, un destin aussi clair qu'absurde. L'œuvre de Camus dans son ensemble, et La Peste en particulier, ressemblerait donc plus à une épopée qu'à une tragédie, puisque le bonheur, bien que limité, reste un état envisageable pour les humains; et Camus d'avouer que «au centre de [son] œuvre, fût-elle noire, rayonne un soleil inépuisable» ${ }^{28}$.

Enfin, dans le contexte global de la création littéraire de Camus, Le Premier Homme apparaît comme une ébauche de la régénération permanente de l'Humanité: un rêve lumineux! Serait-ce la lumière d'une possible rédemption de l'humain? Par la mémoire? Par la sublimation de son passé et de son histoire? Par la révolte positive «qui ne pardonne pas mais qui oublie, réaction ô combien plus fructueuse dans la contingence de l'humain»? En effet, cette tentative de remémoration du passé en guise de réconciliation, avec ses origines et soi-même, serait-elle un signe de la possibilité d'une acceptation tranquille de l'éphémère humain, à condition que l'Humanité réussisse à éliminer ce qui relève de la méchanceté pure? Tout le reste serait, sinon acceptable, du moins supportable pour celui qui réalise enfin sa condition de "bâton qui a deux bouts", rien en-deçà, au-delà rien!

28 A. CAMUS, "L'Été», in Essais, éd. Pléiade, p. 865. 
La soif d'éternité n'est pourtant pas moindre; et «tels que nous sommes braves et fiers et forts ... si nous avions une foi, un Dieu, rien ne pourrait nous entamer» (Camus, 1994: 327); mais y croire apparaît comme un absurde face à la misère, à l'injustice et au crime qui dominent sur terre. L'homme ne serait donc pas un pèlerin en chemin vers la Terre promise, l'Éternité, mais bien un «nomade» dont la vie commence toujours par déménagement et se terminera par évacuation des terres occupées (Ibidem: 328). Inachevé, comme le livre qui lui a emprunté le nom ${ }^{29}$, le "premier homme» le sera toujours. Tous les hommes sont des vies uniques et inachevées; tous les hommes sont des "premiers hommes»; et la seule différence ce sera de "se dire» en quelques mots ou de, "parlant sans cesse et incapable de trouver à travers des milliers de mots» (Ibidem: 352) ce qui pourrait être dit à travers un seul silence, chercher toujours. La quête de l'existence, tout comme la quête littéraire, est inachevable par nature. Être là en révolte permanente contre tout ce qui ne serait pas juste, tout en profitant, au jour le jour, du bonheur prodigué à l'homme entre la terre et le soleil, voilà ce qui serait le propre de l'homme, des hommes, de tous les Meursaults, de tous les Clamence et de tous les Camus; et il faudrait les croire heureux! Et pourquoi pas, car si le mystère du mal, moral ou physique, n'a pas été résolu, il pourrait néanmoins être toléré, à condition que la révolte nous sauve du désespoir, pendant que la solidarité se profile en filigrane à l'horizon.

Avec tant de soleil dans la mémoire, comment ai-je pu parier sur le non-sens? On s'en étonne, autour de moi; je m’en étonne aussi parfois. Je pourrais répondre, et me répondre, que le soleil justement m’y aidait et que sa lumière, à force d'épaisseur, coagule l'univers et ses formes dans un éblouissement obscur. Mais cela peut se dire autrement et je voudrais, devant cette clarté blanche et noire qui, pour moi, a toujours été celle de la vérité, m’expliquer simplement sur cette absurdité que je connais trop pour supporter qu'on en disserte sans nuances. Parler d'elle au demeurant, nous mènera de nouveau au soleil ${ }^{30}$.

29 Remarquons que s'il est vrai que Camus n'a pas pu terminer la rédaction du Premier Homme, il semblerait que son projet était justement de nous donner un récit inachevé:

"Le livre doit être inachevé. Ex.: "Et sur le bateau qui le ramenait en France..." (A. CAMUS, 1994: 333).

30 A. CAMUS, "L'été», in Essais, éd. Pléiade, p. 861. 
En effet, devant le caractère définitif de la mort, voulant éclairer l'absurdité de la vie humaine, Camus, tout en repoussant les solutions moyennes, fait de son intransigeance la solution même. Face au tragique fondé sur la souffrance et la mort, une seule issue: dire oui à la vie comme à cette lumière du sud; autant dire la consécration de l'homme, pourtant fragile et périssable, de l'amour de la vie, non moins condamnée à l'échec, mais dans un élan de communion viscérale avec la terre chaude, la mer immense et la lumière qui les unit à l'immensité du ciel. Lumières d'Afrique ou lumières de l'esprit, c'est tout le chemin de Camus, parcours qui se dessine au fil de sa plume! Lumières du ciel ou du sens, ombres de douleurs physiques ou de l'esprit, font un tout chez Camus. Ce «fulgurant soleil d'Afrique qui traque impitoyablement les ombres» (Roblès, 1964: 57) traduit la lutte que mena Camus pendant toute sa vie. Ayant connu et vécu l'absurde aux côtés de sa génération, sans pouvoir lui échapper, il prône cependant une issue pour l'homme, la seule issue possible selon lui: la révolte solidaire ${ }^{31}$. «L'homme est périssable. Il se peut; mais périssons en résistant, et si le néant nous est réservé, ne faisons pas que ce soit une justice ${ }^{32}$.

31 Il s'agit là peut-être de la vraie «énigme heureuse» (A. CAMUS, "LÉnigme", in Essais, éd. Pléiade, p. 861.) qui, à défaut de comprendre, nous aiderait au moins à accepter notre condition ici, sub sole.

32 Épigraphe tirée d'Oberman, citée par Camus dans Lettres à un ami allemand (IV ${ }^{\mathrm{ème}}$ Lettre), apud, R. GRENIER, 1987: 166.

Senancour, qui publia Oberman en 1804, avait connu l'exil (il est parti en Suisse en 1789); et l'exil avait créé chez lui le sentiment non seulement d'être profondément étranger au monde de son époque, mais aussi d'être définitivement «un étranger», tel que nous le trouvons défini chez Camus. 


\section{BIBLIOGRAPHIE}

\section{Corpus:}

CAMUS Albert (1942a 2006), Le Mythe de Sisyphe, éditions Gallimard. , $\left(1942^{\mathrm{b}}\right)$, L'Étranger, éditions Gallimard. , (2006 - 2008), Euvres Complètes: L'envers et l'endroit (1937); Noces à Tipasa (1939); Caligula (1941); Le Malentendu (1944); La Peste (1947); L’Homme révolté (1951), La Chute (1956); Essais ("L'été à Alger", "Le désert", "L’Énigme"), Paris, éditions de La pléiade. , (1955), «Lettre à Roland Barthes», 11 juillet 1955. , (1958, 2002), Chroniques algériennes 1939 -1958 Actuelles III, Paris, éditions Gallimard. (1962), Carnets (Mai 1935 - Février 1942), Paris, éditions Gallimard.

_, (..., 1994, 2007), Le Premier Homme, éditions Gallimard.

\section{Études:}

BEY, Maïssa (2006), L'ombre d'un homme qui marche au soleil: Réflexions sur Albert Camus, préface de Catherine Camus, Paris, Éditions Chèvre-feuille étoilée.

CAMUS, Catherine, MAHASELA, Marcelle (2010), Albert Camus: Solitaire et solidaire, Paris, éd. Michel Lafon.

CHABOT, Jacques (2002), Albert Camus, la pensée de midi, sl., éditions Édisud.

COMTE-SPONVILlE, André (1995, réed. 2001), Camus, de l'absurde à l'amour, avec des lettres inédites d'Albert Camus. Éditions Paroles d'Aube, réed. La Renaissance du Livre.

CORBIC, Arnaud (2007), Camus et l'homme sans Dieu, Paris, Éditions du Cerf.

DANIEL, Jean (2006), Avec Camus: Comment résister à l'air du temps, Paris, éd. Gallimard.

GUÉRIN, Jean-Yves (coord.) (1985), Camus et la politique: Actes du colloque de Nanterre, 5 - 7 juin 1985.

(2009), Dictionnaire Albert Camus, Paris, Robert Laffont.

GRENIER, Roger (1987), Albert Camus, soleil et ombre, Paris, éd. Gallimard.

LENZINI, José (2009), Les derniers jours de la vie d'Albert Camus, éd. Actes sud.

LOTTAMN, Herbert R. (1978), Albert Camus, traduit de l'Américain par Marianne Véron, Éditions du Seuil, Paris.

PAYETTE, Jean-François, et olivier, Lawrence (dir), (2007), Camus, nouveaux regards sur sa vie et son œuvre, éditions Presses de l'université du Québec.

ROBLÈs, E. (1964), Camus, éd. Hachette, Paris.

TODD, Olivier (1996, 1999), Albert Camus, une vie, Paris, éditions Gallimard. 